\title{
Rapid identification of microorganisms from platelet concentrates by matrix-assisted laser desorption ionization time-of-flight mass spectrometry after short-term incubation on liquid medium
}

\author{
Yasmine Chetouane, ${ }^{1,2}$ Gregory Dubourg, ${ }^{1,2}$ Pierre Gallian, ${ }^{2,3}$ Christophe Flaudrops, ${ }^{1,2}$ \\ Jacques Chiaroni, ${ }^{4}$ Eric Chabrière, ${ }^{1,2}$ Didier Raoult, ${ }^{1,2}$ and Laurence Camoin-Jau (iD) ${ }^{1,2,5}$
}

BACKGROUND: Platelets (PLTs) are especially affected by the risk of bacterial contamination. Matrixassisted laser desorption ionization time-of-flight (MALDI-TOF) is an accurate method for the routine identification of bacterial isolates in microbiology laboratories. We directly applied the MALDI-TOF method to bacterial detection in PLTs. In this study, we evaluated the sensitivity, specificity, and speed of a direct MALDITOF approach compared to the conventional method BACTEC.

STUDY DESIGN AND METHODS: Eight bacteria associated with PLT contamination, cited by the ISBT on transfusion-transmitted infectious diseases, were spiked into PLTs for a final concentration of approximately 100 CFU/bag ( $n=5$ for each strain). The PLTs were then agitated for 24 hours. One milliliter of PLTs was incubated in a shaker incubator for 8 hours at $37^{\circ} \mathrm{C}$ with $1 \mathrm{~mL}$ of trypticase soy broth (TSB). The spectra were analyzed using the MALDI Biotyper software. As a control, $8 \mathrm{~mL}$ of PLTs incubated into BACTEC bottles and a positive bottle were subcultured to ensure identification of bacterial growth.

RESULTS: Regardless of the strain of PLTs tested, MALDI-TOF analysis made detection and early identification possible at 8 hours. Analysis by BACTEC of PLTs infected with Escherichia coli, Bacillus cereus, and Providencia stuartii made early identification possible. For the remaining bacteria, the detection time by BACTEC was significantly longer than 8 hours. CONCLUSION: We demonstrated the possibility of detecting bacteria in PLTs using a standardized culture step in TSB with MALDI-TOF, regardless of the strain, with the same specificity and analytical sensitivity and with a time to results of 12 hours. This direct method presented rapid and reliable results.
$\mathrm{T}$ ransfusion-transmitted bacterial infection (TTBI) of platelet (PLT) concentrates is considered to be associated with significant morbidity and continues to be a problem around the world. ${ }^{1}$ Although different strategies have been implemented to reduce this risk, reports of TTBI continue to emerge. ${ }^{2}$

The occurrence of severe infection has been correlated with the extent of the bacterial proliferation in the

ABBREVIATIONS: MALDI-TOF $=$ matrix-assisted laser desorption ionization time-of-flight; MS = mass spectrometry; TSB = trypticase soy broth; TTBI = transfusion-transmitted bacterial infection.

From the ${ }^{1}$ Aix-Marseille Université, Unité de Recherche sur les Maladies Infectieuses et Tropicales Emergentes (URMITE), UM63, CNRS 7278, IRD 198, INSERM U1095; and ${ }^{2}$ IHU Méditerranée Infection, Pôle des Maladies Infectieuses, Assistance Publique-Hôpitaux de Marseille, Marseille, France; ${ }^{3}$ Etablissement Français du Sang (EFS), La Plaine Saint-Denis, France; and the ${ }^{4}$ Etablissement Français du Sang Alpes Méditerranée and Aix-Marseille Université, CNRS, EFS Biologie des Groupes Sanguins, ADES UMR 7268; and the ${ }^{5}$ Assistance Publique Hôpitaux de Marseille, Laboratoire d'Hématologie, CHU Timone, Marseille, France.

Address reprint requests to: Laurence Camoin-Jau, Unité de Recherche sur les Maladies Infectieuses et Tropicales Emergentes, Aix-Marseille Université, UM63, CNRS 7278, IRD 198, INSERM 1095, IHU - Méditerranée Infection, 19-21 Boulevard Jean Moulin, 13385 Marseille Cedex 05, France; e-mail: laurence. camoin@univ-amu.fr

This work was supported by the Institut HospitaloUniversitaire (IHU) Méditerranée Infection, Marseille.

Received for publication May 6, 2017; revision received October 30, 2017; and accepted October 31, 2017.

doi:10.1111/trf.14430

(C) $2017 \mathrm{AABB}$

TRANSFUSION 2018;58;766-773 
PLT component and is reported for a bacterial load of more than $10^{5}$ colony-forming units (CFU)/mL in PLT concentrates at the time of transfusion. ${ }^{3}$ Donor skin flora, donor asymptomatic bacteria, and contamination during product processing are sources of bacterial contamination. ${ }^{4}$ According to Montag, ${ }^{5}$ initial contamination levels are 10 to $100 \mathrm{CFU}$ per PLT unit (0.03-0.3 CFU/mL). However, storing PLTs at between 20 and $24^{\circ} \mathrm{C}$, with agitation, favors bacterial growth. Nevertheless, bacterial growth kinetics vary considerably, making detection difficult, particularly if the bacteria are in a lag phase. These factors often result in the presence of very low bacterial concentrations shortly after collection, requiring the use of highly sensitive screening methods. ${ }^{6}$ Various detection methods based on culture systems or nucleic acid amplification methods, fluorescence-activated cell sorting, or immunologic detection methods (such as the Pan Genera Detection system), are available. ${ }^{7-9}$

Three automated culture methods have been validated for PLT quality control and are implemented in most European and North American countries: the BacT/ ALERT system (bioMérieux), the BACTEC (BD Diagnostics), and the VersaTrek system (Trek Diagnostics). ${ }^{10}$ The cultivation method with early sampling, applying the "negative-to-date" concept of PLT release, has been implemented in several transfusion facilities. ${ }^{11}$

While the BacT/ALERT system is the most widely used, ${ }^{4}$ comparable performance between BACTEC and BacT/ALERT has been described. The BACTEC system has demonstrated detection of 10 to $50 \mathrm{CFU}$ per culture vial. ${ }^{12}$ Other authors have shown that the system can detect 1 to $10 \mathrm{CFU} / \mathrm{mL}^{13,14}$ However, several false-negative test results have been reported in the Netherlands ${ }^{15}$ and in the United States, ${ }^{16}$ and various studies have confirmed this problem. ${ }^{14,17}$

In recent years, matrix-assisted laser desorption ionization time-of-flight (MALDI-TOF) mass spectrometry (MS) has become a powerful and rapid tool for identifying cultured bacterial isolates. ${ }^{18}$ This method has been extended to the identification of bacteria directly from positive culture broths, with some success. ${ }^{19-22}$ Using MALDI-TOF directly on positive cultures has significantly reduced the mean time to identification. ${ }^{23}$

The aim of this study was to evaluate the performance of MALDI-TOF for the direct identification of pathogens at 8 hours of incubation time. This approach offers major advantages, such as a small volume of PLTs (only $1 \mathrm{~mL}$ ); cost-effectiveness; and a rapid, standardized result, whatever the strain.

\section{MATERIALS AND METHODS}

\section{Microorganism strains}

According to the recommendations of the ISBT working party on transfusion-transmitted infectious diseases, ${ }^{24}$ eight species from the CSUR (Collection de Souches de l'Unité des Rickettsies) collection were tested: Staphylococcus epidermidis (CSUR P2203), Streptococcus pyogenes (CSUR P576), Klebsiella pneumoniae (CSUR P2205), Escherichia coli (CSUR P2202), Staphylococcus aureus (CSUR P2188), Providencia stuartii (CSUR P2204), Pseudomonas aeruginosa (CSUR P2201), and Bacillus cereus (CSUR 329). The bacteria reference strains were stored before use at $-80^{\circ} \mathrm{C}$.

Bacterial suspensions were obtained from cultures placed overnight on 5\% sheep blood agar plates (bioMérieux). The number of CFUs was divided by the product of the dilution factor. Serial dilutions of these suspensions yielded concentrations from which small aliquots were inoculated.

\section{Preparation of PLTs}

The PLTs were provided by the French national blood bank and were obtained after standard processing of whole blood units from healthy, volunteer blood donors. The protocol was approved by the ethics committee of IHU Méditerranée Infection (Reference 2016-002). Whole blood units $(467 \pm 7 \mathrm{~mL})$ were collected on Day 0 with a quintuple bag (DGR7542, Fenwal) and stored overnight at $21 \pm 3^{\circ} \mathrm{C}$. On Day 1, the whole blood was centrifuged at $5.312 \times g$ (acceleration 9, break 5) using a centrifuge (Cryofuge 6000, Heraeus). The centrifuged products were then separated on automatic presses (Compomat G4, NPBI Fresenius), with top-and-bottom separation. Five ABO isogroup buffy coats and $280 \mathrm{~mL}$ PLT additive solutions (InterSol, Fenwal) were pooled using the integrated pooling system in the kit. Pools were then placed in the automatic device, which processes six buffy coat pools at the same time. Each cycle allows simultaneous centrifugation, separation, and leukoreduction of products within 12 minutes. The mean PLT content under routine conditions for TACSI LR-BCPC is $4.5 \pm 0.410,{ }^{11}$ with a mean volume of $333 \pm 11 \mathrm{~mL}$. After preparation, PLTs were stored between 20 and $24^{\circ} \mathrm{C}$, with agitation.

\section{In vitro contamination of PLT units}

Before contamination, the baseline sterility of 40 bags of PLTs was confirmed by microbiologic controls in accordance with the standard operating procedure used in our laboratory. In addition, $8 \mathrm{~mL}$ of sample was inoculated in both aerobic and anaerobic (BacT/ALERT SN; bioMérieux) culture bottles and incubated for up to 7 days.

For inoculation of 40 bags of PLTs, the eight bacterial reference species solution was serially diluted in sterile water to reach a final concentration of approximately 100 $\mathrm{CFU} / \mathrm{mL}$. The inoculate concentrations were quantified in duplicate by direct plating of $1 \mathrm{~mL}$ onto Colombia blood agar plates, incubating them at $37^{\circ} \mathrm{C}$ and enumerating after 24 hours, and were then identified according to our 


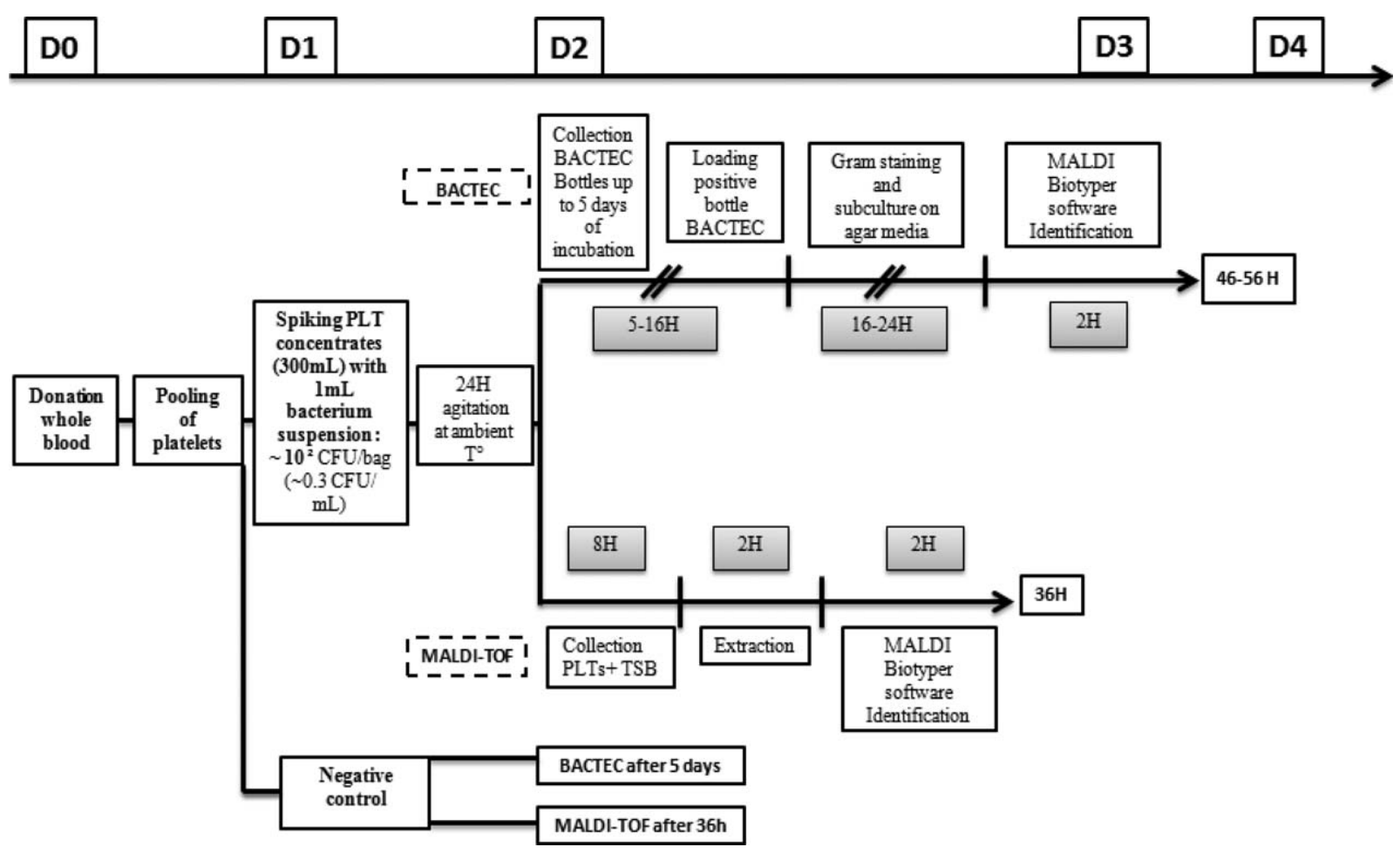

Fig. 1. Study flow chart of PLT inoculation and sampling. $\mathrm{T}^{\circ}=$ temperature.

procedure using mass spectrometric methods (i.e., MALDI-TOF MS, Bruker Daltonics).

One milliliter of inoculum of each bacterial suspension strain was spiked into each bag with a sterile syringe to obtain a final concentration of approximately $0.3 \mathrm{CFU} /$ $\mathrm{mL}$. After contamination, the 40 bags of PLTs were stored under standard blood bank storage conditions for 24 hours with agitation at $50 \mathrm{turn} / \mathrm{min}$ in PLT incubator bags at ambient temperature, according to US Food and Drug Administration recommendations. ${ }^{2}$

\section{Bacterial detection methods}

The flow chart for the study is given in Fig. 1 .

\section{MALDI-TOF procedure}

After agitation for 24 hours in a temperature-controlled environment of $22 \pm 2^{\circ} \mathrm{C}, 1 \mathrm{~mL}$ from each of the 40 spiked bags of PLTs was placed in a 2-mL Eppendorf sterile tube with $1 \mathrm{~mL}$ of trypticase soy broth (TSB) and incubated in a shaker incubator at approximately $50 \mathrm{turn} / \mathrm{min}$ at $37^{\circ} \mathrm{C}$ for 8 hours. Before beginning protein extraction, samples were also taken for determination of bacterial concentration. Briefly, 10-fold dilutions were prepared in TSB, and 0.1-mL samples from each dilution were plated in duplicate onto blood agar plates. Culture plates were incubated at $37^{\circ} \mathrm{C}$ for 24 hours followed by colony counting. The positive PLT sample was then added to $300 \mu \mathrm{L}$ of saponin solution (Sigma-Aldrich) with $700 \mu \mathrm{L}$ of water. The lysate was then thoroughly mixed. The tube was centrifuged

\begin{tabular}{|c|c|c|}
\hline \multicolumn{3}{|c|}{$\begin{array}{l}\text { TABLE 1. Specificity and sensibility of the MALDI- } \\
\text { TOF method* }\end{array}$} \\
\hline & Infected & Not infected \\
\hline Positive test & $\begin{array}{l}\text { (True-positive value) } \\
\text { TP }=1200\end{array}$ & $\begin{array}{l}\text { (False-positive value) } \\
\mathrm{FP}=0\end{array}$ \\
\hline Negative test & $\begin{array}{l}\text { (False-negative value) } \\
\text { FN }=0 \\
\text { Sensitivity }=\mathrm{TP} / \mathrm{TP}+\mathrm{FN} \\
=1200 / 1200+0 \\
=1\end{array}$ & $\begin{array}{l}\text { (True-negative value) } \\
\text { TN }=1200 \\
\text { Specificity }=\text { TN/TN }+ \text { FP } \\
=1200 / 1200+0 \\
=1\end{array}$ \\
\hline $\begin{array}{l}\text { * Thirty rep } \\
\text { unseeded) }\end{array}$ & $\begin{array}{l}\text { tes of each bag of } \\
\text { ted by MALDI-TOF. }\end{array}$ & PLTs (40 seeded and \\
\hline
\end{tabular}

at $16.2 \times g$ for 5 minutes and the supernatant was discarded. The pellet was further washed with $1 \mathrm{~mL}$ of distilled water and the supernatant was discarded after centrifugation at $16.2 \times g$ for 5 minutes. The supernatant was again discarded, and the pellet was air-dried for 10 minutes at room temperature. Subsequently, the supernatant was carefully removed by pipetting and was resuspended with $30 \mu \mathrm{L}$ of formic acid. An equal volume of acetonitrile was then added, followed by centrifugation at $16.2 \times g$ for 5 minutes. The supernatant $(1 \mu \mathrm{L})$ was then spotted onto target slides for MALDI-TOF analysis. One milliliter of 40 other unseeded bags of PLTs followed the same steps previously described.

\section{Spectral analysis and MALDI-TOF MS identification}

The average spectral profiles obtained were analyzed and compared using a computer program (Flex Analysis, 




Fig. 2. Representative MALDI-TOF spectra after PLT contamination with seven bacterial strains. The first spectrum (A) is a negative control. Representative MS spectra of $S$. aureus (B), E. coli (C), S. pyogenes (D), K. pneumoniae (E), P. aeruginosa (F), S. epidermidis (G), and P. stuartii (H), PLTs were spiked with $100 \mathrm{CFU} / \mathrm{bag}$. One milliliter of the spiked PLTs was incubated with TSB and MALDI-TOF analysis was performed after 8 hours' incubation. a.u. = arbitrary units; $\mathbf{m} / \mathbf{z}=$ mass-to-charge ratio. Additional peaks due to the presence of bacteria. [Color figure can be viewed at wileyonlinelibrary.com]

Bruker Daltonics). Identification scores were assigned using the following scoring variables: a score of 1.9 or more indicated species identification, a score of 1.7 to 1.9 indicated genus identification, and a score of less than 1.7 indicated no identification. Samples were considered to be correctly identified by MALDI-TOF when two spectra had a score of 1.9 or more. ${ }^{18}$

\section{Reproducibility of MALDI-TOF MS identification}

To assess the reproducibility of MALDI-TOF MS identification, every strain $(n=8)$ studied was tested five times (five different bags). For every condition, 30 different spots were loaded on the MALDI-TOF plate, giving a total of 1200 MALDI-TOF MS protein profiles derived from each strain. For the negative control, for each of 40 unseeded bags of PLTs, 30 different spots were loaded on the MALDI-TOF plate, giving a total of 1200 MALDI-TOF MS protein profiles.

\section{BACTEC 9240 system analysis and bacterial identification}

Twenty-four hours postinoculation, quantitative cultures were similarly performed by placing $0.1 \mathrm{~mL}$ of PLTs onto $5 \%$ sheep blood agar plates and incubated at $37^{\circ} \mathrm{C}$ for 24 hours. All cultures were performed in duplicate and the counts were averaged.

At the same time, an aliquot of $8 \mathrm{~mL}$ of spiked PLTs was inoculated with culture medium in an aerobic 




Fig. 3. Biotyper score values (mean \pm SD) of spiked PLTs using MALDI-TOF analysis. PLTs were spiked with approximately 100 CFU/bag and MALDI Biotyper software obtained after 24 hours' agitation and 8 hours' incubation for each strain, in triplicate with $1 \mathrm{~mL}$ of PLT samples, with trypticase soy growth medium at $37^{\circ} \mathrm{C}$, testing 30 replicates. Experiments were performed for each strain using five different bags of PLTs. ${ }^{* * *} \mathrm{p}<0.005$ was considered significant.

BACTEC bottle. The culture bottles were incubated in the BACTEC 9240 culture system at $37^{\circ} \mathrm{C}$ for 5 days (three replicates/bag). The signals were recorded continuously to detect any potential changes in response to increasing $\mathrm{CO}_{2}$ production by bacteria. The positive broth sample was subcultured on agar medium and incubated at $37^{\circ} \mathrm{C}$ for 24 hours. Resultant microorganisms were identified by conventional microbiologic methods using MALDI-TOF MS (Bruker Daltonics). ${ }^{18}$

\section{Statistical analysis}

Statistical analysis was performed using computer software (GraphPad Prism 6, GraphPad, Inc.). Descriptive statistics were expressed as mean $\pm \mathrm{SD}$. An analysis of variance was used for intergroup comparisons of the MALDI-TOF data.

Sensitivity and specificity were calculated. "Sensitivity" is the probability that the diagnostic instrument indicates a positive result for individuals with disease (here, positive samples), and "specificity" is the probability of a negative result of the instrument for those patients without the disease.

Comparison of growth rates among bottles was performed for all tested bacteria using McNemar's test for the BACTEC system. The results were evaluated within the $95 \%$ confidence interval and a p value of less than 0.005 was accepted as significant.

\section{RESULTS}

\section{Detection and identification of bacterial contamination of PLTs by MALDI-TOF}

The final concentrations of the bacterial inoculum as determined by quantitative culture are summarized in Table 1 . The range is between 97 and $108 \mathrm{CFU} / \mathrm{mL}$.
The analysis of spectral profiles of spiked PLTs was performed after 24 hours of agitation of the PLTs and 8 hours of incubation in TSB (Fig. 1). As shown in Fig. 2, specific profiles for each strain tested were observed. The mean score for the negative control PLTs obtained with MALDI Biotyper software was $1.13 \pm 0.14$, and never exceeded 1.7 (Fig. 3).

For the eight bacterial strains, the MALDI Biotyper score was always significantly higher than negative controls $(\mathrm{p}<0.005,30$ replicates for each strain and for each bag). The strains tested were correctly identified by MALDI Biotyper at the species level. Indeed, matching scores were all between 1.9 and 2.42 (Fig. 3).

These results showed that for bacterial concentrations in PLTs, MALDI-TOF MS can detect bacterial contamination after only 8 hours of incubation in TSB, after 2 hours of protein extraction, and less than 2 hours of detection by MALDI-TOF for 48 samples whatever the strain. Also, correct identification of the microorganisms was systematically performed with this approach in a fixed time scale of 36 hours (Fig. 1). The specificity of our MALDI-TOF method was $100 \%$ (Table 1).

\section{Detection of bacterial contamination of PLTs by BACTEC}

Table 2 shows the mean values for length of time to detection obtained for each strain, after 24 hours of agitation with a sampling volume of $8 \mathrm{~mL}$. P. aeruginosa (CSUR P2201), S. epidermidis (CSUR P2203), K. pneumoniae (CSUR P2205), and S. pyogenes (CSUR P576) have a longer growing time, with mean detection times, respectively, of 8 hours $56 \pm 0.003$ minutes, 9 hours $41 \pm 0.09$ minutes, 10 hours $29 \pm 0.02$ minutes, and 15 hours $2 \pm 0.02$ minutes ( $\mathrm{p}<0.005, \mathrm{n}=5$ bags for each strain). 


\begin{tabular}{|c|c|c|c|c|}
\hline Microorganisms & $\begin{array}{l}\text { Bacterial inoculums } \\
(\text { CFU/mL) }\end{array}$ & $\begin{array}{l}\text { Times to detection } \\
\text { (hr) of aerobic PLT culture } \\
\text { positivity in BACTEC } \\
\text { bottles ( } n=5 \text { bags) } \dagger\end{array}$ & $\begin{array}{l}\text { Mean titration } \\
\text { (CFU/mL) after } \\
24 \mathrm{hr} \text { of agitation }\end{array}$ & $\begin{array}{c}\text { Mean titration } \\
(\mathrm{CFU} / \mathrm{mL}) \text { after } 24 \mathrm{hr} \text { of } \\
\text { agitation and } 8 \mathrm{hr} \text { of incubation }\end{array}$ \\
\hline \multicolumn{5}{|l|}{ Gram-negative } \\
\hline E. coli & 97 & $4.25(3.34-5.04)$ & $5.60 \times 10^{4}$ & $8.00 \times 10^{5}$ \\
\hline P. stuartii & 99 & $6.38(4.56-7.49)$ & $1.04 \times 10^{4}$ & $1.51 \times 10^{5}$ \\
\hline K. pneumoniae & 101 & $10.29(9.46-10.62)$ & $3.60 \times 10^{3}$ & $3.41 \times 10^{4}$ \\
\hline$P$. aeruginosa & 101.3 & $8.56(8.56-10.03)$ & $4.10 \times 10^{3}$ & $2.77 \times 10^{5}$ \\
\hline \multicolumn{5}{|l|}{ Gram-positive } \\
\hline S. aureus & 106.5 & $8.10(5.43-10.14)$ & $1.30 \times 10^{4}$ & $2.15 \times 10^{5}$ \\
\hline S. epidermidis & 104.2 & $9.41(10.03-14.26)$ & $1.25 \times 10^{3}$ & $3.20 \times 10^{4}$ \\
\hline B. cereus & 108 & $3.41(3.35-4.45)$ & $3.63 \times 10^{4}$ & $4.5 \times 10^{5}$ \\
\hline S. pyogenes & 102.3 & $15.02(14.56-15.3)$ & $1.01 \times 10^{3}$ & $1.17 \times 10^{4}$ \\
\hline
\end{tabular}

Only B. cereus, E. coli, and P. stuartii, which grew rapidly, were detected early, respectively, at 3 hours $41 \pm 0.07$ minutes, 4 hours $25 \pm 0.03$ minutes, and 6 hours $38 \pm 0.04$ minutes. However, a period of 24 hours after the time to detection is necessary to correctly identify the strain by MALDI-TOF. In this case, depending on the strain, 46 or 56 hours were necessary for final identification (Fig. 1).

During this study, no false-negative cases were found, regardless of the method used. On the same days as sampling for BACTEC cultures was performed, titration experiments were performed after 24 hours of agitation and after 8 hours of incubation with TSB. Results of this experiment are shown in Table 2.

\section{DISCUSSION}

In this study, we evaluated the performance of a new strategy: combining a short culture step and MALDI-TOF analysis to detect bacterial contamination of PLTs, with bacteria at approximately $100 \mathrm{CFU} / \mathrm{bag}$ to mimic real-life conditions. We demonstrated that MALDI-TOF methods, combined with the analysis of protein spectra, enabled detection of all the strains tested within a systematically short time period of 8 hours, using only $1 \mathrm{~mL}$ of PLTs sampled for a lower bacterial concentration (approx. 100 $\mathrm{CFU} / \mathrm{bag}$ or $0.3 \mathrm{CFU} / \mathrm{mL}$ ).

In the past few years, MALDI-TOF MS has increasingly been introduced into diagnostic microbiology laboratories for rapid bacterial species identification when sampled directly from bacterial colonies and has allowed bacterial identification directly from positive bottle cultures. To our knowledge, MALDI-TOF MS has never been used to detect bacterial contamination of PLT units. The major advantage of this method compared to currently available techniques is that it combines a standardized time scale for results of sufficient sensitivity.
Under our analysis conditions (approx. $100 \mathrm{CFU} / \mathrm{bag}$ or $0.3 \mathrm{CFU} / \mathrm{mL}$ at inoculation), all bacterial contaminations were detected and identified by standard pattern against the MALDI Biotyper database after 8 hours of incubation and 2 hours of protein extraction. The identification of Gram-negative and Gram-positive bacteria using the direct MALDI-TOF method can be used efficiently to identify bacteria in a positive sample of PLTs. The BACTEC method, which is considered as a reference method, presents a similar sensitivity, ${ }^{14}$ although several falsenegative and false-positive test results have been described. ${ }^{25,26}$

In our model, we chose to use $100 \mathrm{CFU}$ at inoculation. This dose of inoculant, given the few studies that have been carried out, is estimated to have a bacterial load on the day of collection (D0) of between 1 and 65 CFU/bag. ${ }^{27,28}$ This concentration is much lower in the beginning and increases until it reaches the threshold of $10^{5} \mathrm{CFU} / \mathrm{mL}$, considered by health authorities as the threshold representing a vital risk for the patient. ${ }^{29,30}$

In addition, only $1 \mathrm{~mL}$ of PLTs was used for the MALDI-TOF analysis to preserve the majority of PLTs for transfusion. To increase the limit of detection of our method, an incubation step at $37^{\circ} \mathrm{C}$, with stirring, in the presence of TSB was added. This protocol detected all bacteria within 8 hours, for a final bacterial concentration of approximately $100 \mathrm{CFU} / \mathrm{bag}(0.3$ $\mathrm{CFU} / \mathrm{mL}$ ), with the same sensitivity and specificity as BACTEC.

In contrast, $8 \mathrm{~mL}$ of PLTs was used for BACTEC analysis, in accordance with the CLSI (Clinical and Laboratory Standards Institute) guidelines, because the number of microorganisms present in PLTs may be relatively small. ${ }^{31}$ Also, the inoculum volume strongly affects culture sensitivity and incubation time requirements. 
Moreover, our results were obtained over short periods, within 24 hours of bag preparation. Wagner and Robinette $^{32}$ in their evaluation of the BacT/ALERT system, concluded that sampling times of 24 hours after preparation would be necessary to have confidence in detecting bacteria in PLTs.

Analysis of the time to results clearly demonstrated a reduction in the turnaround time for identification when performing direct identification with the MALDI-TOF extraction method compared to the conventional method using BACTEC. Indeed, the BACTEC method requires subculturing a positive culture onto solid medium before identification by MALDI-TOF.

Also, our method of combining a short incubation in liquid medium and analysis by MALDI-TOF allowed all strains tested to be identified within a shorter time. In practice, time to identification of bacterial contamination may naturally have a major impact on the prevention and outcomes of TTBI.

Our method met our objectives. In addition to the sensitivity and specificity criteria, it satisfied the early detection criteria.

Among the limitations of our study are the relatively small numbers of PLT units, because this is a muchrequested product. Therefore, the feasibility of the MALDI-TOF MS test should be demonstrated in a routine clinical setting. Other studies should be carried out to evaluate whether the technique performs as well with apheresis PLTs and PLTs, the conservation solution of which is $100 \%$ plasma.

For the routine test procedure, after collecting samples between 24 and 36 hours after blood donation, inoculation with the TSB medium for 8 hours is carried out, followed by extraction and identification by MALDI-TOF, for duration of 4 hours, as proposed for BacT/ALERT and BACTEC. The extraction step can even be automated to further reduce the time required to obtain results. In conclusion, MALDI-TOF MS may prove to be a method for rapidly detecting bacteria, compared to BACTEC, for bags with an initial concentration of approximately $100 \mathrm{CFU}$. Our method, combining a short incubation in a liquid medium and analysis by MALDI-TOF, demonstrated its ability to rapidly detect contaminated PLT units and presented major advantages in combining early results with sufficient sensitivity, regardless of the strain, compared to BACTEC.

\section{ACKNOWLEDGMENTS}

The authors thank the volunteer blood donors. JC, PG, DR, and LCJ designed the study, performed data acquisition, analyzed and interpreted the data, and drafted the manuscript; YC and CF performed the MALDI-TOF detection; YC and LCJ interpreted the data and wrote, edited, and revised the manuscript; and all authors approved the submitted final version.

\section{CONFLICT OF INTEREST}

The authors have disclosed no conflicts of interest.

\section{REFERENCES}

1. Hong H, Xiao W, Lazarus HM, et al. Detection of septic transfusion reactions to platelet transfusions by active and passive surveillance. Blood 2016;127:496-502.

2. Bacterial detection testing by blood collection establishments and transfusion services to enhance the safety and availability of platelets for transfusion [Internet]. Silver Spring (MD): U.S. Department of Health and Human Services, Food and Drug Administration, Center for Biologics Evaluation and Research; 2016 [cited 2015 Nov 20]. Available from: http://www.fda.gov/downloads/BiologicsBloodVaccines/ GuidanceComplianceRegulatoryInformation/Guidances/ Blood/UCM425952.pdf.

3. Blajchman MA, Goldman M, Baeza F. Improving the bacteriological safety of platelet transfusions. Transfus Med Rev 2004;18:11-24.

4. Pietersz RN, Reesink HW, Panzer S, et al. Bacterial contamination in platelet concentrates. Vox Sang 2014;106:256-83.

5. Montag T. Strategies of bacteria screening in cellular blood components. Clin Chem Lab Med 2008;46:926-32.

6. Walther-Wenke G, Schrezenmeier H, Deitenbeck R, et al. Screening of platelet concentrates for bacterial contamination: spectrum of bacteria detected, proportion of transfused units, and clinical follow-up. Ann Hematol 2009;89:83-91.

7. Störmer M, Vollmer T. Diagnostic methods for platelet bacteria screening: current status and developments. Transfus Med Hemother 2014;41:19-27.

8. Schmidt M, Sireis W, Seifried E. Implementation of bacterial detection methods into blood donor screening-overview of different technologies. Transfus Med Hemother 2011;38: 259-65.

9. Muller TH, Mohr H, Montag T. Methods for the detection of bacterial contamination in blood products. Clin Chem Lab Med 2008;46:933-46.

10. Dunne WM, Case LK, Isgriggs L, et al. In-house validation of the BACTEC 9240 blood culture system for detection of bacterial contamination in platelet concentrates. Transfusion 2005;45:1138-42.

11. De Korte D, Curvers J, de Kort WL, et al. Effects of skin disinfection method, deviation bag, and bacterial screening on clinical safety of platelet transfusions in the Netherlands. Transfusion 2006;46:476-48.

12. Roh KH, Kim JY, Kim HN, et al. Evaluation of BACTEC Plus aerobic and anaerobic blood culture bottles and BacT/Alert FAN aerobic and anaerobic blood culture bottles for the detection of bacteremia in ICU patients. Diagn Microbiol Infect Dis 2012;73:239-42.

13. Riedel S, Siwek G, Beekmann SE, et al. Comparison of the BACTEC 9240 and BacT/Alert blood culture systems for detection of bacterial contamination in platelet concentrates. J Clin Microbiol 2006;44:2262-4. 
14. Benjamin RJ, Wagner SJ. The residual risk of sepsis: modeling the effect of concentration on bacterial detection in twobottle culture systems and an estimation of false-negative culture rates. Transfusion 2007;47:1381-9.

15. Boekhorst PA, Beckers E, Vos MC, et al. Clinical significance of bacteriologic screening in platelet concentrates. Transfusion 2005;45:514-9.

16. Eder AF, Dy BA, Barton J, et al. The American Red Cross Hemovigilance Program: advancing the safety of blood donation and transfusion. Immunohematology 2009;25: 179-85.

17. Lessa F, Leparc GF, Benson K, et al. Fatal group C streptococcal infection due to transfusion of a bacterially contaminated pooled platelet unit despite routine bacterial culture screening. Transfusion 2008;48:2177-83.

18. Seng P, Rolain JM, Fournier PE, et al. MALDI-TOF-mass spectrometry applications in clinical microbiology. Future Microbiol 2010;5:1733-54.

19. Kok J, Thomas LC, Olma T, et al. Identification of bacteria in blood culture broths using matrix-assisted laser desorptionionization Sepsityper and time of flight mass spectrometry. PLoS One 2011;6:e23285.

20. Buchan BW, Riebe KM, Ledeboer NA. Comparison of the MALDI Biotyper system using Sepsityper specimen processing to routine microbiological methods for identification of bacteria from positive blood culture bottles. J Clin Microbiol 2012;50:346-52.

21. Jamal W, Saleem R, Rotimi VO. Rapid identification of pathogens directly from blood culture bottles by Bruker matrixassisted laser desorption laser ionization-time of flight mass spectrometry versus routine methods. Diagn Microbiol Infect Dis 2013;76:404-8.

22. Martiny D, Dediste A, Vandenberg O. Comparison of an inhouse method and the commercial Sepsityper kit for bacterial identification directly from positive blood culture broths by matrix-assisted laser desorption-ionisation time-of-flight mass spectrometry. Eur J Clin Microbiol Infect Dis 2012;31: 2269-81.
23. Schneiderhan W, Grundt A, Worner S, et al. Work flow analysis of around-the-clock processing of blood culture samples and integrated MALDI-TOF mass spectrometry analysis for the diagnosis of bloodstream infections. Clin Chem 2013;59: 1649-56.

24. Störmer M, Arroyo A, Brachert J, et al. Establishment of the first international repository for transfusion-relevant bacteria reference strains: ISBT working party transfusiontransmitted infectious diseases (WP-TTID), subgroup on bacteria. Vox Sang 2012;102:22-31.

25. Benjamin RJ, Dy B, Perez J, et al. Bacterial culture of apheresis platelets: a mathematical model of the residual rate of contamination based on unconfirmed positive results. Vox Sang 2014;106:23-30.

26. Kou Y, Pagotto F, Hannach B, et al. Fatal false-negative transfusion infection involving a buffy coat platelet pool contaminated with biofilm-positive Staphylococcus epidermidis: a case report. Transfusion 2015;55:2384-9.

27. Pearce S, Rowe GP, Field SP. Screening of platelets for bacterial contamination at the Welsh Blood Service. Transfus Med 2010;21:25-32.

28. Murphy WG, Foley M, Doherty C, et al. Screening platelet concentrates for bacterial contamination: low numbers of bacteria and slow growth in contaminated units mandate an alternative approach to product safety. Vox Sang 2008;95: 13-9.

29. Jacobs MR, Good CE, Lazarus HM, et al. Relationship between bacterial load, species virulence, and transfusion reaction with transfusion of bacterially contaminated platelets. Clin Infect Dis 2008;46:1214-20.

30. Wagner SJ. Transfusion-transmitted bacterial infections: risks, sources and interventions. Vox Sang 2004;86:157-63.

31. Principles and procedures for blood cultures; approved guideline. CLSI document M47-A. Wayne (PA): Clinical and Laboratory Standards Institute; 2007.

32. Wagner SJ, Robinette D. Evaluation of an automated microbiological blood culture device for detection of bacteria in platelet components. Transfusion 1998;38:674-8. 